\title{
Kidneys preservation before transplantation
}

\section{Mini-review}

Any method of preservation has the objective to maintain the organ in the best conditions during the period between the retrieval and the transplant, lowering the ischemia/reperfusion injury. Ideal preservation system should allow extending the ischemia time of the graft, improving its function and enabling the use of organs which initially were considered not suitable for transplant. Static storage with hypothermic solution (perfusing the organ through a catheter positioned in the renal artery with a cold storing solution immediately after the retrieval, leaving the graft inside a sterile container covered with ice with a temperature within $0^{\circ} \mathrm{C}$ and $4^{\circ} \mathrm{C}$ ) is still considered the gold standard for kidneys preservation. ${ }^{1}$ Plenty of perfusion solutions exist, with different chemical-physical characteristics; those which mostly have been used for kidneys preservation are Belzer UW® Cold Storage Solution (UW Solution)and Celsior ${ }^{\circledR}$ Genzyme. Comparative trials among these perfusion solutions have not showed advantages in using the first or the second, in particular any difference in terms of delay graft function (DGF) and graft and patient survival. ${ }^{2-5}$ Despite recent studies have showed that other preservation methods allow better results after transplantation, the simplicity (application capacity even by non-expert personnel) and the economicity (few devices needed) of the static storage still justify its widespread utilization. ${ }^{6}$

Because of the shortage of the number of the donors, kidney transplants are performed often and often using grafts coming from marginal donors (ECD, extended criteria donors). ${ }^{7}$ As these grafts are more susceptible to the ischemia injury, ${ }^{8}$ these organs have higher rate of post-transplant complications, in particular DGF and primary non function (PNF) ${ }^{8-12}$ Strategies to improve the outcome of transplants performed using grafts coming from "non-ideal" donors are therefore investigated. Some authors showed that hypothermic pulsatile perfusion significantly reduces the risk of DGF compared to the classic static storage. ${ }^{13-15}$ Despite it is a significant difference, the rate of DGF observed using the pulsatile perfusion is not absolutely much inferior than that one observed in the static storage. This type of perfusion is ensured positioning the kidney after the retrieval inside a machine (LifePort Kidney Transporter ${ }^{\circledR}-$ Organ Recovery System), which continually perfuses the kidney with an hypothermic solution with a pulsatile pressure through a peristaltic pump within a closed circulation, measuring peristaltic pulse rate, solution temperature, mean systolic and diastolic pressures, real time flow and flow resistance. Hypothermic pulsatile perfusion would have the possibility to provide to the tissues higher level of ATP and to perfuse continually microcirculation, eliminating in this way waste products.

Other strategies to reduce the ischemia/reperfusion and to improve the function of the graft are the normal temperature (or sub-normal) preservation systems. The normothermic perfusion (EVNP, ex vivo normothermic perfusion) and the resurrection techniques have already been used for heart and lung transplantation, ${ }^{16-18}$ but just recently they have been applied also to the kidney transplantation. ${ }^{19}$ In an experimental porcine model of EVNP kidneys undergone to this treatment had lower level flow resistance, better homeostasis of the acid-basis equilibria, high level of oxygen consumption, lower tubular

\author{
Volume 4 Issue 4 - 2017

\section{Lorenzo Maroni, Matteo Ravaioli, Antonio Daniele Pinna}

Department of Medical and Surgical Sciences, University of Bologna, Italy

\section{Correspondence: Matteo Ravaioli, Department of Medical and Surgical Sciences, University of Bologna, Bologna, Italy,} Email matteo.ravaioli@aosp.bo.it

Received: June 12, 2017 | Published: June 16, 2017

injury, high level of IL-6 and of heat shock protein 70, compared to control group kidneys (static storage), with no differences in inflammatory cytokines (IL- $1 \beta$, IL-8, TNF- $\alpha$ ) expression levels or in kidney function (creatinine clearance).$^{20}$ Other clinical studies shew that EVNP reduces rates of DGF of transplants performed using grafts coming from ECD, without modifying outcome in the long run (graft and patient survival). ${ }^{21}$ Actual data from the literature report single centers experiences, with a limited number of treated patients, and no multicentric study with a wide sample study is available.

The last research sector to improve the function of the kidney to transplant concerns the hypothermic oxygenated perfusion. The use of oxygenated solution would have the goal to provide oxygen to the organ, so to support the re-synthesis of ATP, delaying the formation of many toxic intracellular substances. However high concentrations of oxygen increase the production of reactive oxygen species (ROS), which cause further oxidative stress and tissue injury. ${ }^{22,23}$ The oxygenation of the solution and of the organ can be effectuated through a lot of techniques: adding oxygen to the perfusion solutions (static or dynamic), increasing the atmospheric pressure (hyperbaric oxygenation, static or dynamic), using oxygen artificial transporters. We still don't know if organs oxygenation could offer advantages to the outcome after kidney transplant, ${ }^{24}$ several trials which try to answer to this query are ongoing. Despite technological progress drive the research and the clinical practice to the application of new strategies, the majority of the Transplant Centers still mostly uses static storage. Pulsatile perfusion has allowed to reduce some short term outcomes, without modifying graft or patient survival and without allowing the use of kidneys initially considered not suitable for transplant. Kidneys oxygenation (normothermic of hypothermic) would provide good future perspectives, able to improve sensitively short and long term outcomes and to increase the pool of grafts to destine to transplantation.

\section{Acknowledgements}

None.

\section{Conflict of interest}

The author declares no conflict of interest. 


\section{References}

1. Hosgood SA, Yang B, Bagul A, et al. A comparison of hypothermic machine perfusion versus static cold storage in an experimental model of renal ischemia reperfusion injury. Transplantation. 2010;89(7):830-837.

2. Faenza A, Catena F, Nardo B, et al. Kidney preservation with university of wisconsin and Celsiorsolution: a prospectivemulticenterrandomizedstudy. Transplantation. 2001;72(7):1274-1277.

3. Montalti R, Nardo B, Capocasale E, et al. Kidney transplantation from elderlydonors: a prospectiverandomizedstudycomparingcelsior and UW solutions. Transplant Proc. 2005;37(6):2454-2455.

4. Nunes P, Mota A, Figueiredo A, et al. Efficacy of renal preservation: comparative study of celsior and university of wisconsin solutions. Transplant Proc. 2007;39(8):2478-2479.

5. Tillou X, Collon S, Surga N, et al. Comparison of UW and Celsior: longterm results in kidney transplantation. AnnTransplant. 2013;18:146-152.

6. Catena F, Coccolini G, Montori C, et al. Kidney preservation: review of present and future perspective. TransplProc. 2013;45(9):3170-3177.

7. Womer KL, Kaplan B. Recent developments in kidney transplantation - a critical assessment. Am J Transplant. 2009;9(6):1265-1271.

8. Metzger RA, Delmonico FL, Feng S, et al. Expanded criteria donors for kidney transplantation. Am J Transplant. 2003;3(Suppl.4):114-125.

9. Kuo PC, Johnson LB, Schweitzer EJ, et al. Utilization of the older donor for renal transplantation. Am J Surg. 1996;172(5):556-557.

10. Hariharan S, McBride MA, Benett LE, et al. Risk factors for renal allograft survival from older cadaver donors. Transplantation. 1997;64(12):17481754.

11. Ojo AO, Hanson JA, Meier-Kriesche H, et al. Survival in recipients of marginal cadaveric donor kidneys compared with other recipients and wait-listed transplant candidates. J Am Soc Nephrol. 2001;12(3):589-597.

12. Irish WD, Ilsley JN, Schnitzler MA. A risk prediction model for delayed graft function in the current era of deceased donor renal transplantation. Am J Transplant. 2010;10(10):2279-2286.
13. Moers C, Smits JM, Maathuis MH. Machine perfusion or cold storage in deceased-donor kidney transplantation. N Engl J Med. 2009;360(1):7-19.

14. Treckmann J, Moers C, Smits JM, et al. Machine perfusion versus cold storage for preservation of kidneys from expanded criteria donors after brain death. TransplInt. 2011;24(6):458-554.

15. Stratta RJ, Moore PS, Farney AC, et al. Influence of pulsatile perfusion preservation on outcomes in kidney transplantation from expanded criteria donors. J Am Coll Surg. 2007;204(5):873-882.

16. Tenderich G, Tsui S, El-Banayosy A. The 1-year follow-up of the PROTECT patient population using the organ care system. J Heart Lung Transplant. 2008;27(S2):S166.

17. McCurry K, Jeevanandam V, Mihaljevic T. Prospective multicenter safety and effectiveness evaluation of the organ care system device of cardiac use (PROCEED). J Heart Lung Transplant. 2008;27(2):S166.

18. Steen S, Sjöberg T, Pierre L, Liao Q, et al. Transplantation of lungs from a non-heart-beating donor. Lancet. 2001;357(9259):825-829.

19. Hosgood SA, Nicholson ML. First in man renal transplantation after ex vivo normothermic perfusion. Transplantation. 2011;92(7):735-738.

20. Hosgood SA, Patel M, Nicholson ML. The conditioning effect of ex vivo normothermic perfusion in an experimental kidney model. J Surg Res. 2013;182(1):153-160

21. Nicholson ML, Hosgood SA. Renal transplantation after ex vivo normothermic perfusion: the first clinical study. Am J Transplant. 2013;13(5):1246-1252.

22. Fuller BJ, Lee CY. Hypothermic perfusion preservation: The future of organ preservation revisited? Cryobiology. 2007;54(2):129-145.

23. Mitchell T, Saba H, Laakman J. Role of mitochondrial-derived oxidants in renal tubular cell cold-storage injury. Free RadicBiol Med. 2010;49(8):1273-1282.

24. Hosgood SA, Nicholson HF, Nicholson ML. Oxygenated kidney preservation techniques. Transplantation. 2012;93(5):455-459. 